\title{
Preface
}

\section{Alexander Vasil'ev}

Published online: 12 April 2011

(c) The Author(s) 2011. This article is published with open access at Springerlink.com

An important feature of the development in natural sciences during the last decades is the increasing degree of cross-fertilization between mathematics and physics with greatly enriching both subjects. Analysis and geometry are one of the most relevant parts of mathematics for the study of classical and quantum mechanics and field theories. Besides, new developments in physics have inspired mathematics and led to the birth of new mathematical disciplines. Mathematics is, on the other hand, united by its methodological standards and rigor, and complements physics insights this way. However, there still exists a gap in understanding of physical reasoning and motivation among contemporary analysts and differential geometers. And conversely, practitioners in physics sometimes use analysis and geometry as a tool and do not follow in-depth the intrinsic mathematical developments.

Efforts within the research community are taken to fill this gap. Such efforts have been continuously supported by the European and US research and funding institutions as well as by several national research councils. The creation of formal, or informal scientific networks is particularly important in this process in which scientists combine their efforts by organizing conferences, workshops, and research exchanges, and by promoting further collaborative work and training of young promising researchers in creative interdisciplinary environments. A new jet of ideas gushed out around 2000 at a crossroad of analysis and mathematical physics. The challenge of structural understanding of non-equilibrium interface dynamics has become increasingly important in mathematics and physics. In particular, an elegant description of 2D conformally invariant statistical physical systems in terms of stochastic (Schramm) Loewner contour evolution implied revolutionary progress at the beginning of XXI century. Laplacian growth, coming from fluid dynamics as a Hele-Shaw problem,

\footnotetext{
A. Vasil'ev ( $\square)$

Department of Mathematics, University of Bergen, 5020 Bergen, Norway

e-mail: alexander.vasiliev@math.uib.no
} 
became a rapidly developing field of mathematical physics after discovery of its relation to exactly solvable models. These development and few related ideas resulted in a network that has been formed in the last decade. It is only natural that the publishing activities of researchers of this community resulted in some special volumes and issues such as a special Birkhäuser volume 'Analysis and Mathematical Physics' [1] and a special issue of the Journal 'Complex Analysis and Operator Theory' [2]. After the series of publications, our efforts have culminated in the appearance in 2011 of a new Birkhäuser Journal, 'Analysis and Mathematical Physics', which publishes current research results as well as selected high-quality survey articles in real, complex, harmonic, geometric analysis originating and/or having applications in mathematical physics. The Journal's main goal is promoting the dialog between specialists in these areas.

We hope that it will be of interest for specialists and graduate students specializing in mathematics and/or mathematical physics. We especially appreciate the continuous help of the Birkhäuser Verlag publishing company that makes our dreams a reality.

Open Access This article is distributed under the terms of the Creative Commons Attribution Noncommercial License which permits any noncommercial use, distribution, and reproduction in any medium, provided the original author(s) and source are credited.

\section{References}

1. Gustafsson, B., Vasil'ev, A. (eds.): Analysis and Mathematical Physics. Trends in Mathematics. Birkhäuser Verlag, Basel, pp. 514 (2009)

2. Gustafsson, B., Vasil'ev, A. (eds.): Complex Analysis and Mathematical Physics. Complex Analysis Operation Theory, vol. 4, no. 3, pp. 229 (2010) 